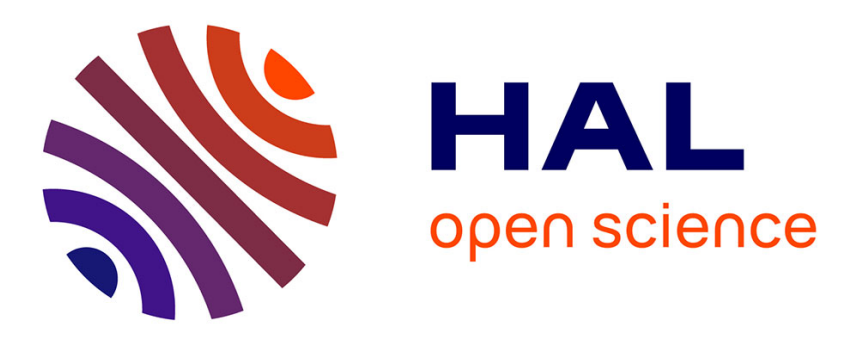

\title{
Collective oscillations of proteins proven by terahertz spectroscopy in aqueous medium
}

Yoann Meriguet, Mathias Lechelon, Matteo Gori, Ilaria Nardecchia, Frederic

Teppe, Anastasiia Kudashova, Dominique Coquillat, Luca Varani, Marco

Pettini, Jeremie Torres

\section{To cite this version:}

Yoann Meriguet, Mathias Lechelon, Matteo Gori, Ilaria Nardecchia, Frederic Teppe, et al.. Collective oscillations of proteins proven by terahertz spectroscopy in aqueous medium. 2019 44th International Conference on Infrared, Millimeter, and Terahertz Waves (IRMMW-THz), Sep 2019, Paris, France. pp.1-2, 10.1109/IRMMW-THz.2019.8873880 . hal-02448214

\section{HAL Id: hal-02448214 \\ https://hal.science/hal-02448214}

Submitted on 28 Feb 2020

HAL is a multi-disciplinary open access archive for the deposit and dissemination of scientific research documents, whether they are published or not. The documents may come from teaching and research institutions in France or abroad, or from public or private research centers.
L'archive ouverte pluridisciplinaire HAL, est destinée au dépôt et à la diffusion de documents scientifiques de niveau recherche, publiés ou non, émanant des établissements d'enseignement et de recherche français ou étrangers, des laboratoires publics ou privés. 


\title{
Collective oscillations of proteins proven by terahertz spectroscopy in aqueous medium
}

\author{
Yoann Meriguet ${ }^{1,2}$, Mathias Lechelon ${ }^{3,4}$, Matteo Gori ${ }^{3}$, Ilaria Nardecchia ${ }^{3}$, Frederic Teppe ${ }^{2}$, Anastasiia \\ Kudashova $^{1}$, Dominique Coquillat ${ }^{2}$, Luca Varani ${ }^{1}$, Marco Pettini ${ }^{3}$, Jeremie Torres ${ }^{1}$ \\ ${ }^{1}$ Institut d'Electronique et des Systemes (IES), University of Montpellier, CNRS, Montpellier, France \\ ${ }^{2}$ Laboratoire Charles Coulomb (L2C), University of Montpellier, CNRS, Montpellier, France \\ ${ }^{3}$ Centre de Physique Theorique (CPT), Aix-Marseille University, CNRS, Marseille, France \\ ${ }^{4}$ Centre d'Immunologie de Marseille-Luminy (CIML), Aix-Marseille University, CNRS, Marseille, France
}

\begin{abstract}
Understanding the forces that enable protein interactions is a major scientific challenge. Indeed, Brownian diffusion alone, which is usually considered as the main engine of protein dynamics, does not explain the rapidity and efficiency of the biomolecular reactions at works in cells. In this work, we will present observations of the collective oscillations of a model protein driven out-of-equilibrium by means of optical excitation in aqueous medium using terahertz spectroscopy.
\end{abstract}

\section{INTRODUCTION}

$\mathrm{B}$ ROWNIAN diffusion of freely moving biomolecules is usually considered to drive the dynamics of the molecular machinery maintaining cellular functions and thus life. However, the high efficiency and rapidity of the encounters between cognate partners of biochemical reactions inside living cells call for a more convincing explanation with respect to purely thermal-fluctuations-driven model of random walks. It has been surmised that a suitable interplay between Brownian diffusion and selective electrodynamic interactions acting at a long-distance one (up to thousands of Angstroms) could significantly accelerate the encounter times of interacting biomolecules in living matter [1]. Collective oscillations of out-of-thermal equilibrium bio-molecule, or of a substantial fraction of its atoms, is essential to generate giant oscillating molecular dipole moments and so, to activate longrange electrodynamic interactions [2]. Up to now, no experimental evidence indicates that these collective oscillations, that should lie in the terahertz $(\mathrm{THz})$ frequency range, are sufficiently intense to activate some interactions between biomolecules. However, since these collective molecular oscillations are accompanied by the creation of a large dipole moment [3], $\mathrm{THz}$ spectroscopy should be able to detect a spectral signature in the absorption coefficient of the proteins and this even in aqueous medium despite the huge absorption provided by the solvent.

Although the existence of collective vibration modes in biomolecules at thermal equilibrium has already been experimentally proven by mean of Raman spectroscopy [4], no experience has made it possible to highlight an out-ofequilibrium collective oscillations.

\section{MATERIALS AND METHODS}

For experiment, a model protein has been chosen: Albumin from Bovine Serum (BSA). BSA, Alexa Fluor 488 (AF488) conjugate was purchased from ThermoFisher (A13100) in lyophilized powder. It was centrifuged to remove supernatant before use. Then BSA-AF488 was added to $\mathrm{NaCl}$ solution at
$250 \mathrm{mM}$ to obtain $1 \mathrm{mg} / \mathrm{ml}$ solution. The dye has excitation/emission of 497/520 $\mathrm{nm}$. Alexa488 fluorochrome, which is covalently bonded at the lysine residues of the BSA is excited by means of an argon laser (wavelength $488 \mathrm{~nm}$ ). Two separated and different experiments, performed in Montpellier (France) and in Rome (Italy), respectively, have been performed to obtain the terahertz nonequilibrium spectra of the model protein chosen. The former used a microwire as a local probe (fig. $1 \mathrm{a}, \mathrm{b}$ ), whereas the latter used a nanorectenna (fig. $1 \mathrm{c}, \mathrm{d}$ ). They have been used at room temperature.

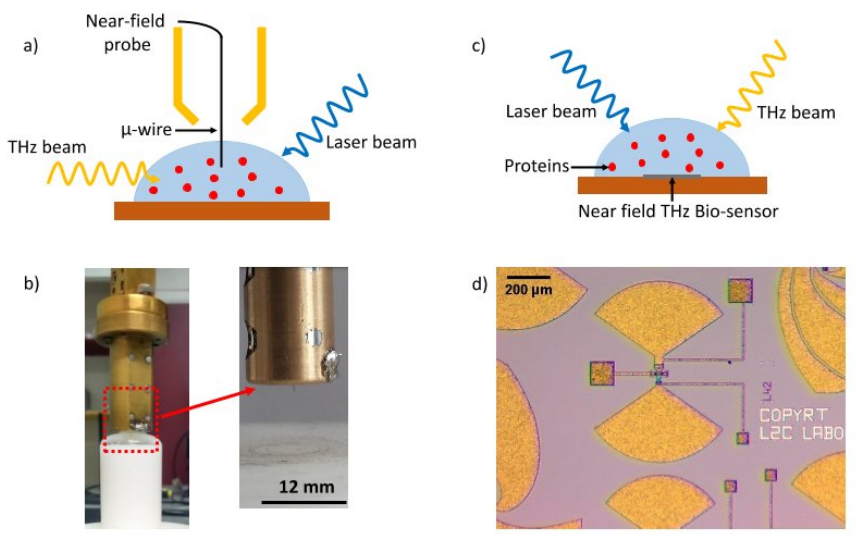

Fig. 1 Experimental setup of THz absorption near-field spectroscopy. (a) A drop of the biological sample placed under the near-field probe, which is directly immersed into the solution. (b) Picture of the near-field probe and its microwire. (c) A drop of the biological sample placed above the near-field rectenna. (d) Microscopy picture of the bow-tie antenna.

\section{RESULTS}

Using two different and complementary $\mathrm{THz}$ near field spectroscopic techniques, we show that such a phenomenon is actually possible. By considering a watery solution of a model protein, the BSA, we found that this protein displays a remarkable absorption feature around $0.314 \mathrm{THz}$ (Fig. 2. a, b, c) when driven in a stationary non-equilibrium state by means of optical pumping [5]. Fig. 2 (a) shows the normalized spectra of BSA, by subtraction of water spectra, without (black) and with (red, blue, green) blue light illumination using the microwire and depending of the duration of illumination (3 to 9 minutes). The curve in absence of illumination has no special variation contrary to illumination curves. Indeed, there was a strong peak appearance at 0.314 THz. This peak increase as a function of the time of illumination. Maximum value was obtained after 9 minutes of illumination. Fig. 2 (b) shows the normalized spectra of BSA, also by subtraction of water spectra, this time obtained by using a rectenna probe. Peak absorption at $0.314 \mathrm{THz}$ appears after 10 and $15 \mathrm{~min}$. Both of the results displayed the same 
absorption peak as we can observe on Fig. 2 (c) which is a comparison of the two normalized spectra for the longest duration.

(a)

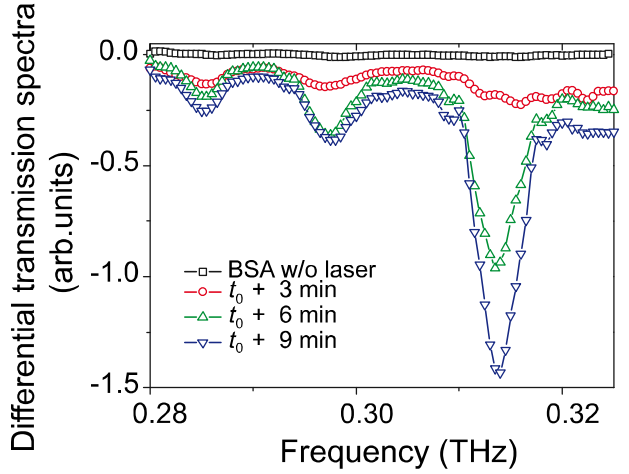

(b)

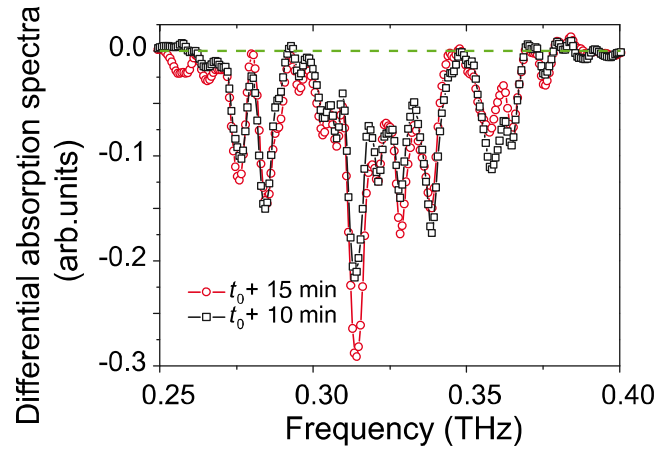

(c)

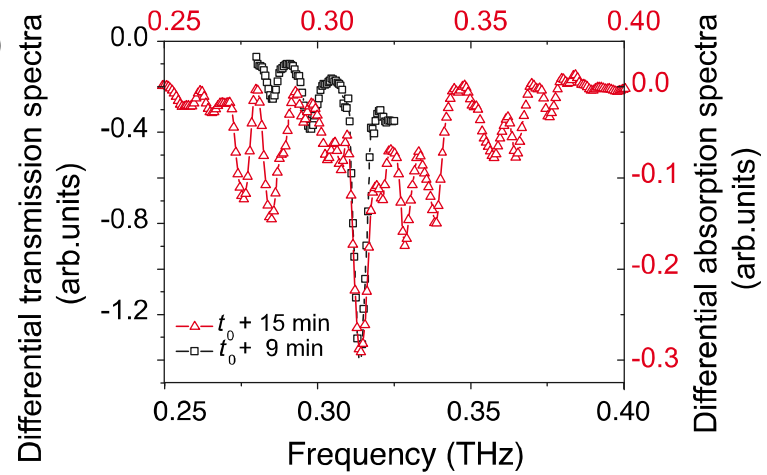

Fig. 2. Differential transmission and absorption spectra as functions of the frequency. (a) Spectra obtained using the microwire probe after subtraction of the water spectrum in the absence of illumination and in the presence of illumination for the reported durations. (b) Spectra obtained using the rectenna, after subtraction of the protein solution without illumi- nation, for the reported durations. (c) Comparison of the two normalized spectra for the longest illumination durations. [5].

Since $\mathrm{THz}$ extinction in water is huge $(2000 \mathrm{~dB} / \mathrm{cm}[6])$, the emergence of this spectral feature of the protein out of the water absorption background must be associated with the activation of a giant dipole moment. This can happen only as a consequence of the activation of a coherent oscillation of the whole molecule, possibly together with a dipole moment strengthening due to an electret-like structuring of water dipoles of the first hydration layers of the proteins, as the hydration shell might contribute to the magnitude of the protein dipole moment.

According to our classical version of the Fröhlich model, it is also expected that the appearance of a collective oscillation should exhibit a threshold-like behaviour when increasing the energy flowing through the protein. Actually, Fig. 5 (a) presents a clear threshold in the intensity of the resonance peak at $0.314 \mathrm{THz}$ when the optical input power exceeds 10 $\mu \mathrm{W}$. By using a classical formalism for the analysis of the outof-equilibrium phonon condensation we have calculated the intensity of the normal vibrational modes of the BSA-protein as a function of the source power injected through the protein. Fig. 5 (b) highlights a threshold-like behaviour of the intensity of the fundamental mode that accumulates the energy at the expenses of the excited modes, in qualitative agreement with the experimental outcome. By increasing the number of modes included in the calculation this threshold becomes more and more evident. The experimental and theoretical results reported in Fig. 5 agree also in displaying a saturation effect occurring at large values of the energy input rate.
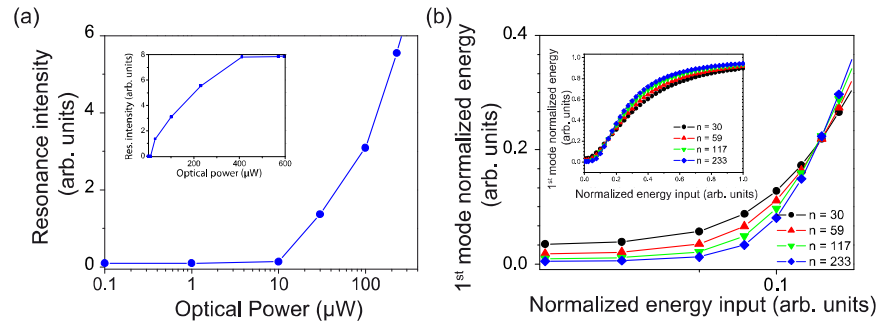

Fig. 3. Threshold-like behaviour of giant dipolar oscillations. (a) Intensity of the resonant peak measured at $0.314 \mathrm{THz}$ as a function of the optical laser power. (b) Normalized energy of the fundamental mode calculated as a function of the normalized source power. The different curves correspond to the reported numbers of normal modes of the BSA protein. Theory and experiment are in qualitative agreement.

The experimental outcomes are in very good qualitative agreement with the theory and in excellent quantitative agreement with a theoretical result allowing to identify the observed spectral feature with a collective oscillation of the entire molecule. We emphasise that this result may open a broad domain of systematic investigations of non-equilibrium activation mechanisms and properties of collective oscillations of different kinds of biomolecules. These interactions could play a relevant role in regulating the molecular machinery at work in living matter.

\section{REFERENCES}

[1]. I. Nardecchia, L. Spinelli, J. Preto, M. Gori, E. Floriani, S. Jaeger, P. Ferrier, and M. Pettini, "Experimental detection of long-distance interactions between biomolecules throught diffusion behavior: Numerical study," Phys. Rev. E, vol. 90, pp. 022703, Aug, 2014.

[2]. H. Fröhlich, "Selective long range dispersion forces between large systems," Physics Letters A, vol. 39, pp. 153-154, 1972.

[3]. J. Preto, M. Pettini, and J. A. Tuszinski, "Possible role of electrodynamic interactions in long-distance biomolecular recognition," Phys. Rev. E, vol. 91, pp. 052710, 2015.

[4]. P. Painter, L. Mosher, and C. Rhoads, "Low frequency modes in the Raman spectra of proteins," Biopolymers, vol. 21, pp. 1469-1472, July, 1982.

[5]. I. Nardecchia, J. Torres, M. Lechelon, V. Giliberti, M. Ortolani, P. Nouvel, M. Gori, Y. Meriguet, I. Donato, J. Preto, L. Varani, J. Sturgis, and M. Pettini, "Out-of-equilibrium collective oscillation as phonon condensation in a model protein," Phys. Rev. X, vol. 8, pp. 031061, 2018.

[6]. P.H. Siegel, "Terahertz technology in biology and medicine," IEEE Trans. Mic. Theo. and Techn., vol. 52, 10, pp. 2438-2447, 2004. 